\title{
GLCM - SVM based Classification of Brain MRI with K-Means Clusters
}

\section{B.Thamaraichelvi}

\begin{abstract}
In this proposed method, MR Brain image segmentation technique based on K-means clustering combined with Discrete Wavelet Transform (DWT) based feature extraction and Gray Level Co-Occurrence Matrix (GLCM) based feature selection approach has been presented. A Perfect Radial Basis Function (RBF) - Support Vector Machine (SVM) Classifier has been selected for this process. The Performance of the classifier was estimated through accuracy based on the fractions selectivity and sensitivity. Accuracy of the proposed classifier was found to be 93\%. Moreover, in this proposed method, instead of selecting the cluster centres in a random manner, Histogram technique was used.

Keywords: Centroid selection, Discrete Wavelet Transforn (DWT), GLCM feature selection, Histon formation, K-means clustering, RBF-SVM Classifier.
\end{abstract}

\section{INTRODUCTION}

Tapas Kanungo et al. (2002) analyzed and implemented the clustering techniques with K-Means for pattern recognition to reduce the distance from the data point to its closest center [1]. Jing- Hao Xue et al.(2003) proposed an integrated method for unsupervised segmentation of Magnetic Resonance brain images. In that method the image was de-noised by using the wavelet filter and segmented with minimum error [2]. Laszlo Szilagyi et al. (2011) explained briefly the combined c- means clustering for the compensation of Intensity Inhomogeneity[3]. Kuntimad et al. (1999) Presented a perfect image segmentation with pulse coupled neural networks [4]. Jussi Tohka et al.(2010) implemented a noval method for the classification of tissues in Magnetic Resonance brain images [5]. Li-Hong Juang and Ming-Ni Wu (2010) introduced tumor objects tracking method for MR Brain images with K-Means clustering [6].

Dongxiang Chi (2011) explained about the SOM$\mathrm{K}$ and SOM- KS segmentation techniques and it is observed that both the methods can achieve better segmentation results with less computational time [7]. In the proposed method k-means clustering approach has been selected due to its low computational complexity.

\section{PROPOSED METHODOLOGY}

In this proposed technique, the input MR Brain image is pre- processed by using median filter, with the help of histogram based Centre initialization algorithm three clusters are formed to extract the Region Of Interest (ROI).

\footnotetext{
Revised Manuscript Received on February 05, 2020.

* Correspondence Author

B.Thamaraichelvi*, Department of Electrical Engineering, Annamalai University, Chidambaram, TamilNadu, India.
}

(C) The Authors. Published by Blue Eyes Intelligence Engineering and Sciences Publication (BEIESP). This is an open access article under the CC BY-NC-ND license (http://creativecommons.org/licenses/by-nc-nd/4.0/)
Then three level DWT technique is used to extract the features in terms of its eigen values and eigen vectors, the Gray Level Co-Occurrence Matrix (GLCM) method is used for its dimensionality reduction and to select the salient features. These selected features are given to Supervised Radial Basis Function - SVM classifier as inputs to identify the given input as either normal or abnormal. The following flowchart (Fig.1) presents the proceeding steps for this work.

\section{CLUSTERING TECHNIQUES}

A. Initialization of centroids:

A Histogram is a powerful technique used with image enhancement and segmentation. It is a graphical representation of the intensity values of pixels in an image. There may be 256 different intensity levels with 8-bit grayscale image and the Histogram of that image gives the details about the distribution of the intensity range of 256 pixels. In this proposed method, the concept of median filtering is used for noise removal by smoothing the intensity variation between a pixel and its nearby. The Histon is constructed as follows, Thamaraichelvi B et al.(2015) [8].

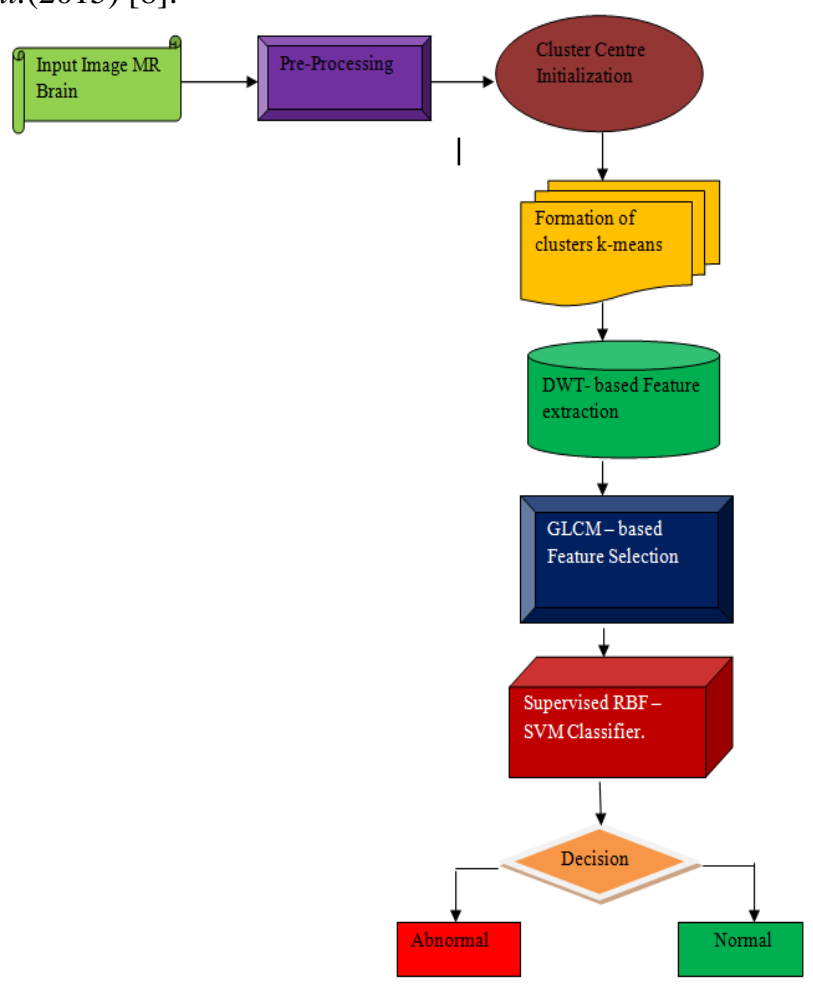

Fig. 1. Schematic diagram for the proposed method 

intensity levels.

\section{B. K-means cluster:} Function is given by,

$H(t)=\sum_{m=1}^{M^{\prime}} \sum_{n=1}^{N^{\prime}}(1+A(m, n)) \delta\left(I^{\prime}(m, n)-t\right), \quad 0 \leq t \leq S-1$

Where, ' $\delta($.$) 'is the impulse function and ' \mathrm{S}$ ' represents the

K-Means clustering is the simplest method with minimum computational complexity. It is an unsupervised method used for bio-medical image segmentation process when the number of clusters is well known Radha et al. (2011) [9]. It is also called as partitioning algorithm. The Objective

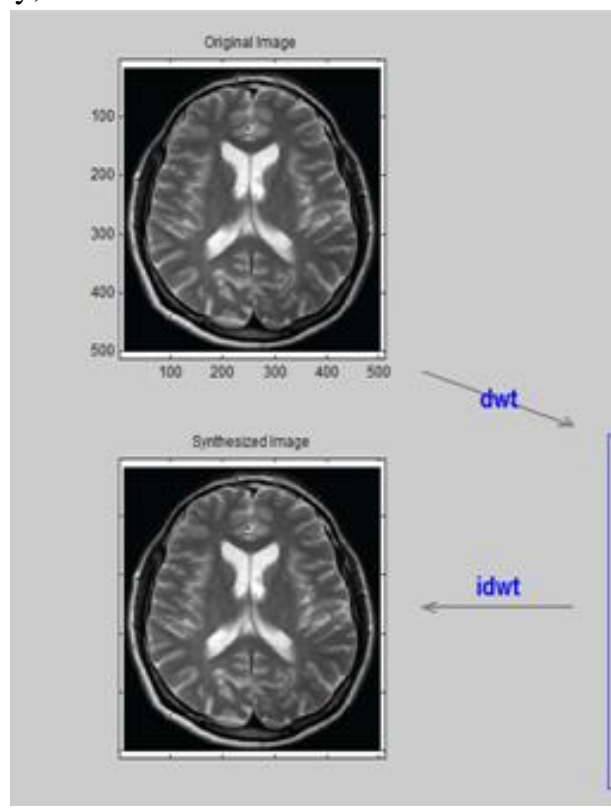

Fig. 2. A Common Method to find 3 Level DWT Decomposition
3. The Euclidean distance from the data point to the mean of each cluster was calculated for all clusters.

4. The data points are allocated to the closest cluster.

5. The process continued till all the data points are allocated to the nearest cluster.

\section{FEATURE EXTRACTION AND SELECTION}

S.Chaplot et al. (2006) explained that in Fourier transform an image has been represented by its frequency contents, but in Wavelet transform an image has been decomposed into different frequency levels with spatially localized [10]. Doubechesis-2 Wavelet in level 3 is selected for this work and the image is of size $512 \times 512$ and $1 \mathrm{~mm}$ thickness each. Fig. 2. shows the 3 level decomposition of the original image.

The Wavelet decomposition technique uses the sub-band LL for the next level. Totally, 4096 coefficients have been extracted from the LL sub-band with the help of db-2 Wavelet tool.

The features are then selected through GLCM method which is a statistical approach used to select the second order textural features. Mohanaiah et al. (2013)

$$
J_{k-\text { means }}=\sum_{r=1}^{R} \sum_{i=1}^{S}\left\|S_{i}-C_{r}\right\|^{2}
$$

Where' $S_{i}$ ' represents data points and ' $C_{r}$ ' means cluster centre, with these concepts, this technique has been considered for this analysis.

\section{K-Means Clustering Algorithm}

1. Cluster centres must be initialized.

2. The data points are divided into a ' $k$ ' number of clusters and also randomly assigned to the clusters.

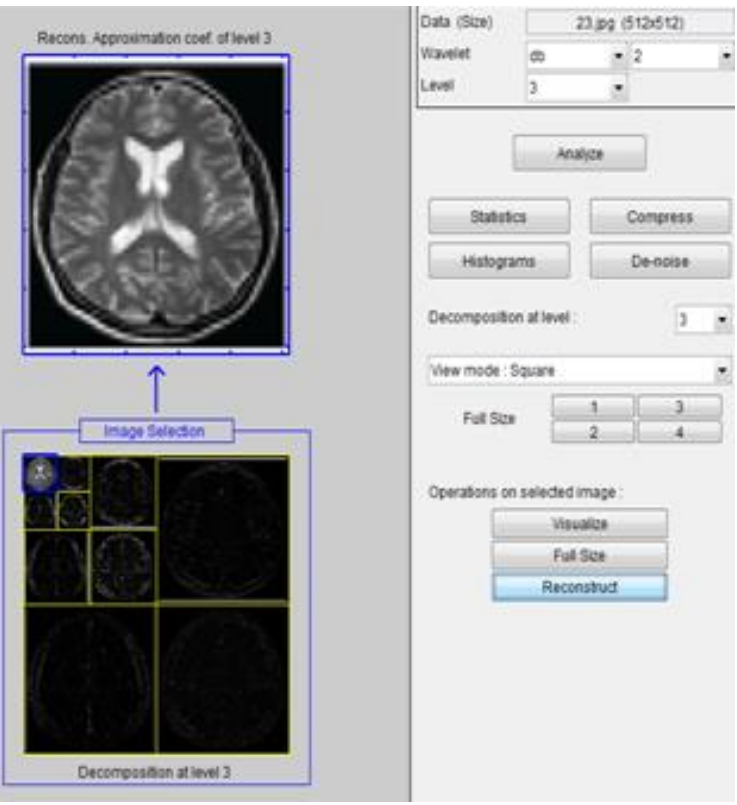

explained about this method mainly used to identify the spatial relationship between a pixel with its immediate neighbouring having a distance ' $d$ ' in the direction ' $\theta$ ' [11]. According to Haralick et al. (1973) the features that can be extracted through GLCM are entropy, correlation, energy, contrast, Mean, Standard -deviation,Variance etc., The selected features are then given to the classifier [12].

\section{RESULT AND DISCUSSION}

The selected image is of size " $512 \times 512$ " and thickness " 1 " $\mathrm{mm}$ in each. The images are preprocessed with the help of median filter. A kernel of size ' $3 \times 3$ ' has been preferred and applied over the entire image to collect the details about the neighbour-hood pixels. Three intensity levels are created for the brain tissues like GM, WM, CSF respectively. The accuracy of the segmentation along with classification was evaluated based on the terms TP,TN,FP,FN. The following figure depicts the outcome of the application of $\mathrm{k}$-means clustering. Fig. 3(i) shows the real input images, fig. 3(ii) gives the outcome of median filtering process and fig. 3(iii)

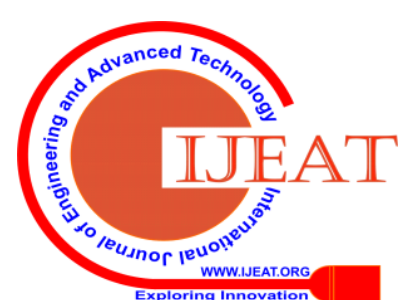


clearly represents the output of k-means clustering for normal brain images. Table 1 . gives the quantitative evaluation of the proposed clustering technique and Table 2. represents the result of glcm based feature selection method for normal brain images. Fig. 4(i) shows the real input images, fig. 4(ii) gives the outcome of median filtering process and fig. 4(iii) clearly represents the outcome of the preferred clustering for abnormal images. Table 3 . gives the evaluation of the selected clustering technique for abnormal MR images. Table 4. represents the result of glcm based feature selection method for abnormal brain images. Totally 40 normal cases and 50 abnormal cases were analysed. The outcome of the preferred RBF- SVM classifier was analysed from the Table 5 and it can detect 47 abnormal and 36 normal cases correctly by giving an accuracy of around $93 \%$.
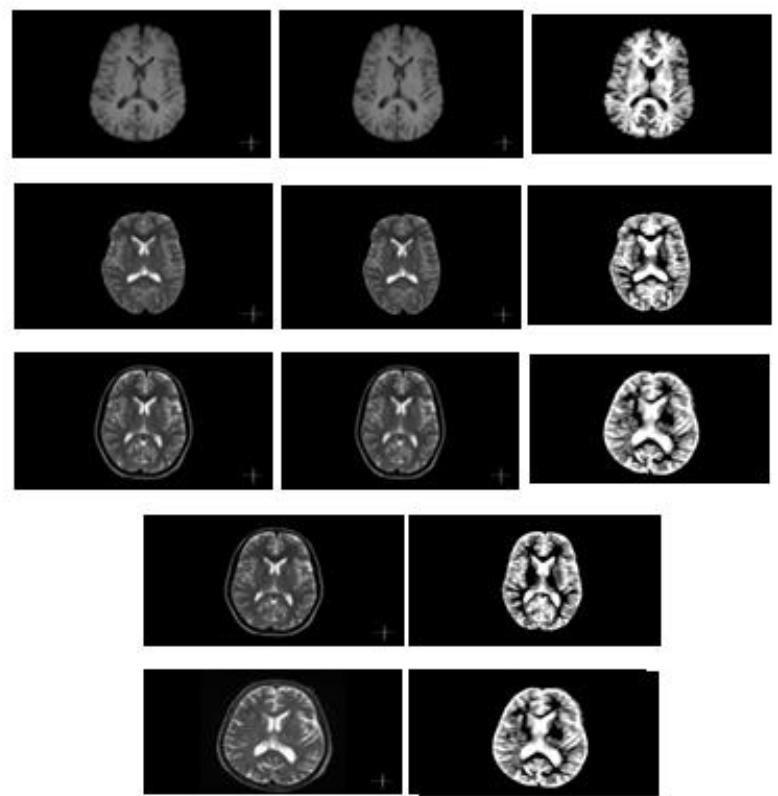

(i)

(ii)

(iii)

Fig. 3. Normal MR Brain Image Analysis (i) Five Real Input Images (ii) Median Filtered Images (iii) Segmented Images.

Table 1. Quantitaive Evaluation for Normal MR Brain Images (iii) K-Means Technique

\begin{tabular}{|c|c|c|c|c|c|c|}
\hline Images & $\begin{array}{c}\text { Sensitivity } \\
\%\end{array}$ & $\begin{array}{c}\text { Specificity } \\
\%\end{array}$ & $\begin{array}{c}\text { Accuracy } \\
\%\end{array}$ & Similarity & $\begin{array}{c}\text { MCR } \\
\%\end{array}$ & $\begin{array}{c}\text { Execution } \\
\text { Time (sec.) }\end{array}$ \\
\hline 1 & 55.41 & 52.28 & 56.88 & 0.4786 & 43.12 & 4.62789 \\
\hline 2 & 59.09 & 56.82 & 57.63 & 0.4956 & 42.37 & 3.54867 \\
\hline 3 & 54.89 & 50.43 & 54.34 & 0.3406 & 45.66 & 3.43372 \\
\hline 4 & 60.35 & 58.72 & 60.52 & 0.3524 & 39.48 & 3.58846 \\
\hline 5 & 65.92 & 59.48 & 63.86 & 0.4956 & 36.14 & 3.07963 \\
\hline
\end{tabular}

Table 2. GLCM based Feature Extraction Method for normal MR Brain Images

\begin{tabular}{|c|c|c|c|c|c|}
\hline $\begin{array}{c}\text { GLCM } \\
\text { Features }\end{array}$ & Data 1 & Data 2 & Data 3 & Data 4 & Data 5 \\
\hline Contrast & 0.85505 & 0.68575 & 0.59655 & 0.79166 & 0.871 \\
\hline
\end{tabular}


GLCM - SVM based Classification of Brain MRI with K-Means Clusters

\begin{tabular}{|c|c|c|c|c|c|}
\hline Correlation & 0.91402 & 0.77256 & 0.85992 & 0.88091 & 0.89269 \\
\hline Energy & 0.13433 & 0.09337 & 0.12157 & 0.12595 & 0.11379 \\
\hline $\begin{array}{c}\text { Standard } \\
\text { deviation }\end{array}$ & 0.16337 & 0.17514 & 0.20064 & 0.16654 & 0.16618 \\
\hline Entropy & 2.5246 & 2.7689 & 2.6457 & 2.5921 & 2.6655 \\
\hline
\end{tabular}

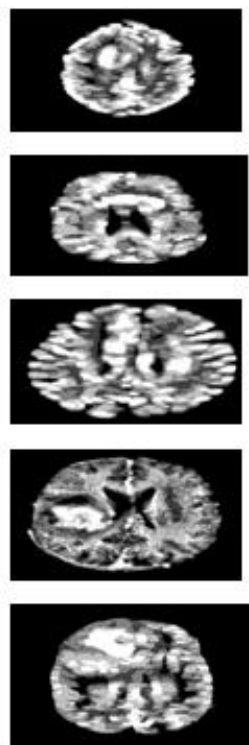

(i)
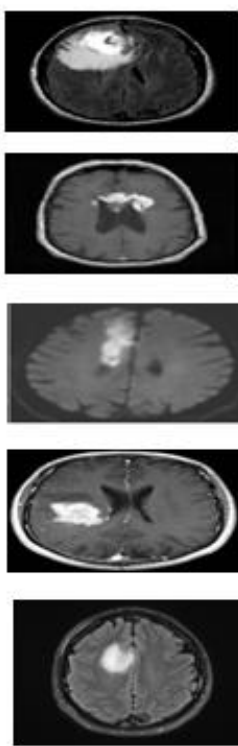

(ii)
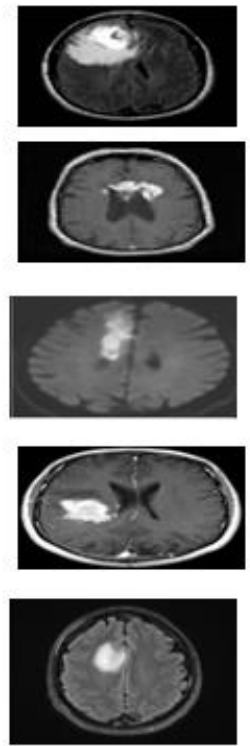

(iii)

Fig. 4. Abnormal MR Brain Image Analysis (i) Five Real Input Images (ii) Median Filtered Images (iii) Segmented Images

Table 3. Performance Evaluation for Abnormal MR Brain Images

(iii) K-Means Technique

\begin{tabular}{|c|c|c|c|c|c|c|}
\hline Images & $\begin{array}{c}\text { Sensitivity } \\
\%\end{array}$ & $\begin{array}{c}\text { Specificity } \\
\%\end{array}$ & $\begin{array}{c}\text { Accuracy } \\
\%\end{array}$ & Similarity & $\begin{array}{c}\text { MCR } \\
\%\end{array}$ & $\begin{array}{c}\text { Execution } \\
\text { Time (sec.) }\end{array}$ \\
\hline 1 & 60.74 & 52.87 & 55.63 & 0.3574 & 44.37 & 3.86939 \\
\hline 2 & 52.69 & 50.93 & 51.02 & 0.3401 & 48.98 & 3.28361 \\
\hline 3 & 63.86 & 59.26 & 61.3 & 0.4391 & 38.7 & 3.25279 \\
\hline 4 & 50.47 & 46.28 & 45.39 & 0.2338 & 54.61 & 3.36634 \\
\hline 5 & 54.83 & 48.24 & 50.08 & 0.2972 & 49.92 & 3.95304 \\
\hline
\end{tabular}

Table 4. GLCM based Feature Extraction Methods for abnormal MR Brain Images

\begin{tabular}{|c|c|c|c|c|c|}
\hline $\begin{array}{c}\text { GLCM } \\
\text { Features }\end{array}$ & Data 1 & Data 2 & Data 3 & Data 4 & Data 5 \\
\hline Contrast & 0.82583 & 0.51278 & 0.50149 & 0.77428 & 0.57393 \\
\hline
\end{tabular}

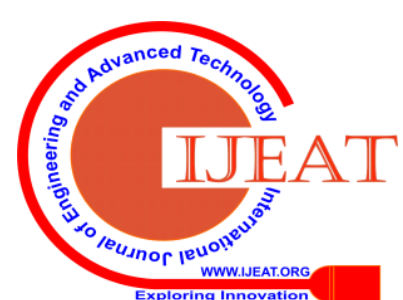




\begin{tabular}{|c|c|c|c|c|c|}
\hline Correlation & 0.91277 & 0.91065 & 0.90111 & 0.93807 & 0.92424 \\
\hline Energy & 0.09358 & 0.09402 & 0.11798 & 0.14076 & 0.29116 \\
\hline $\begin{array}{c}\text { Standard } \\
\text { deviation }\end{array}$ & 0.28153 & 0.21394 & 0.2011 & 0.22587 & 0.23057 \\
\hline Entropy & 2.8531 & 2.7939 & 2.6354 & 2.503 & 2.2396 \\
\hline
\end{tabular}

Table 5 Performance Evaluation of SVM Classifier

\begin{tabular}{|c|c|c|c|c|c|c|c|c|}
\hline Sl.no. & $\begin{array}{c}\text { Classification } \\
\text { Technique }\end{array}$ & $\begin{array}{c}\text { True } \\
\text { positive }\end{array}$ & $\begin{array}{c}\text { True } \\
\text { negative }\end{array}$ & $\begin{array}{c}\text { False } \\
\text { positive }\end{array}$ & $\begin{array}{c}\text { False } \\
\text { negative }\end{array}$ & Sensitivity & Specificity & $\begin{array}{c}\text { Accuracy } \\
(\%)\end{array}$ \\
\hline 1. & RBF- SVM & 47 & 36 & 4 & 3 & 0.94 & 0.925 & $93 \%$ \\
\hline
\end{tabular}

Total Number of Normal Cases 40/ Abnormal Cases 50

\section{CONCLUSION}

The present work is focussed towards the segmentation and classification of brain tissues for tumor deduction. This work includes five stages. Median filtering, clustering, feature extraction, feature selection and classification. This proposed work produces the classification accuracy as around $93 \%$. This work can also be extended to diagnose the diseases in MR brain -images using hybrid combination of swarm evolutionary methods.

\section{ACKNOWLEDGEMENT}

The author is thankful to the Department of Radiology, Raja Muthaiah Medical College and Hospital (RMMCH), Annamalai University, for providing us with the MRI brain data.

\section{REFERENCES:}

1. Tapas Kanungo, David M.M ount, Nathan S.Netanyahu, Christine D.Piatko, Ruth Silverman, Angela Y.Wu,"An Efficient K-Means Clustering Algorithm: Analysis and Implementation", IEEE Transactions on Pattern Analysis and Machine Intelligence, Vol.24, No. 7, pp. 881- 892, 2002.

2. Jing- Hao Xue, Aleksandra Pizurica, Wilfried Philips, Etienne Kerre, Rik Van De Walle, Ignace Lemahieu , "An Integrated Method of Adaptive Enhancement for Unsupervised Segmentation of MRI Brain Images", Pattern recognition letters, Vol.24, pp. 2549-2560, 2003.

3. László Szilágyi, Sándor M. Szilágyi, Balázs Benyó, and Zoltán Benyo, "Intensity Inhomogeneity Compensation and

4. Segmentation of MR Brain Images using Hybrid $C$-Means Clustering Models", Biomedical Signal Processing and Control, Vol. 6, Issue 1, pp. 3-12, 2011

5. 4. Kuntimad, G and Ranganath, H.S, " Perfect Image Segmentation using Pulse Coupled Neural Networks", IEEE Transactions on neural networks, Vol. 10, No.3, pp. 591-598, 1999.

6. Jussi Tohka, Ivo D, Dinov, David W, Shattuck, Arthur W, and Toga, "Brain MRI Tissue Classification Based on Local Markov Random
Fields", Magnetic Resonance Imaging, Vol. 28, Issue 4, pp. 557-573, 2010 .

7. Li-Hong Juang, and Ming-Ni Wu, "MRI Brain lesion Image Detection based on Color-Converted K-Means Clustering Segmentation”, Measurement, Vol. 43, Issue 7, pp. 941-949, 2010.

8. Dongxiang Chi, "Self- Organizing Map- Based Color Image Segmentation with K-Means Clustering and Saliency Map", International Scholarly Research Network Signal Processing, (Research article id- 393891), pp. 1-18, 2011.

9. Thamaraichelvi B and Yamuna G, "A Complexity Reduced FCM Based Segmentation Technique for Brain MRI Image Classification", Journal of Medical Imaging and Health Informatics, Vol. 5, pp.202209, 2015.

10. Radha Chitta, Rong Jin, Timothy C.Havens, Anil K.Jain, "Approximate Kernel K-Means: Solution to Large Scale Kernel Clustering”, KDD'11, August 21-24,SanDiego,California, USA, 2011.

11. Chaplot S, Patnaik L.M and Jagannathan N.R, "Classification of Magnetic Resonance Brain Images using Wavelets as Input to Support Vector Machine and Neural Network", Biomedical signal processing and control, Vol. 1, pp. 86-92, 2016.

12. Mohanaiah P, Sathyanarayana P and Gurukumar L, "Image Texture Feature Extraction using GLCM Approach", International journal of scientific and research publications, Vol.3, Issue5, pp. 1-5, 2013.

13. Haralick R.M, Shanmugam K and Dinstein L, "Textural Features for Image Classification", IEEE Transactions on Systems, Man and Cybernetics, Vol. 3, No. 6, pp. 610-621, 1973.

\section{AUTHORS PROFILE}

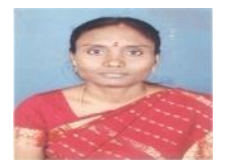

Balan. Thamaraichelvi received her B.E. degree in Electrical and Electronics Engineering (EEE) in the year 1994 from Thiagaraja College of Engineering, Madurai Kamaraj University, TamilNadu, M.E., degree in Process control and Instrumentation Engineering (PC\&I) in the year 2004 and ph.D., degree in Electrical Engineering in the year 2017, from Annamalai University, Chidambaram, TamilNadu,. She has 15 years of teaching experience and she is currently working as an Assistant Professor in the Department of Electrical Engineering at Annamalai university. She has published and presented papers in National and International conferences and journals in the area of Image processing. Her research interest includes Digital signal processing, Image segmentation, Medical Imaging and soft computing. 